\title{
Simulation of a Flexible Manufacturing System with AutoMod Software
}

\author{
Zixia Chen ${ }^{1}$, Changbing Jiang ${ }^{2}$ \\ ${ }^{1}$ Yibin Vocational and Technical College, Yibin, China \\ ${ }^{2}$ Zhejiang Gongshang University, Hangzhou, China \\ E-mail: czx@mail.zjgsu.edu.cn, jcb@mail.zjgsu.edu.cn \\ Received June 2, 2011; revised July 4, 2011; accepted July 15, 2011
}

\begin{abstract}
A flexible manufacturing system (FMS) is a highly automated, complex system. Simulation is a well-proven method to design or analyze an FMS. Deployment of a radio frequency identification (RFID) system in FMS produces large volumes of RFID data streams, which provide valuable information to improve the operation of FMS. Different frameworks are presented in this paper regarding the use of RFID data streams in an FMS simulation. Simulations are performed with AutoMod software. Related technical details are also presented. The paper's structure is complied as the following steps: step 1, the introduction of AMHS, FMS and AutoMod; step 2, FMS simulation using AutoMod software; step 3, simulation frameworks driven by RFID data streams; step 4, conclusion.
\end{abstract}

Keywords: FMS, RFID, Data Stream, Simulation, AutoMod Software

\section{Introduction}

\subsection{AMHS and FMS}

An automated materials handling system (AMHS) stores, retrieves and moves materials through processes to change their form and packaging. It relies primarily on automated devices to handle these materials. AMHS, such as the Automated Storage and Retrieval System (AS/RS) used in the logistics sector or other automated material handling systems utilized in factories play an important role in this aggressively competitive environment $[1,2]$. Therefore, improvements to AMHS are of great value.

The flexible manufacturing system (FMS) plays a more and more important role in AMHS. Figure 1 demonstrates a flexible manufacturing system that processes metal parts. The storage/retrieval machine (S/RM) retrieves pallets of raw parts from the storage racks and places them on conveyors at the end of each aisle. An operator then removes a part from its pallet and fastens it to a fixture on an adjacent conveyor. The conveyor transports the fixtures to a pickup point, where they are loaded onto an automated guided vehicle (AGV).

Six computer numeric control (CNC) machines pro- cess parts in the system. A head changer is also available for drilling the parts. The AGVs transport the fixtures to the required CNCs, or head stations. When all processing of a part has been completed, an AGV returns the part with its fixture to the conveyor near the storage racks. An operator removes the completed part from the fixture and returns it to a pallet for storage on the racks. The empty fixture then circulates on the conveyor to receive a new raw part.

This FMS is a typical discrete event system. While it is difficult to evaluate its efficiency with traditional methods because of random factors and computing complexity, simulation is a well-proven way to design and analyze FMS.

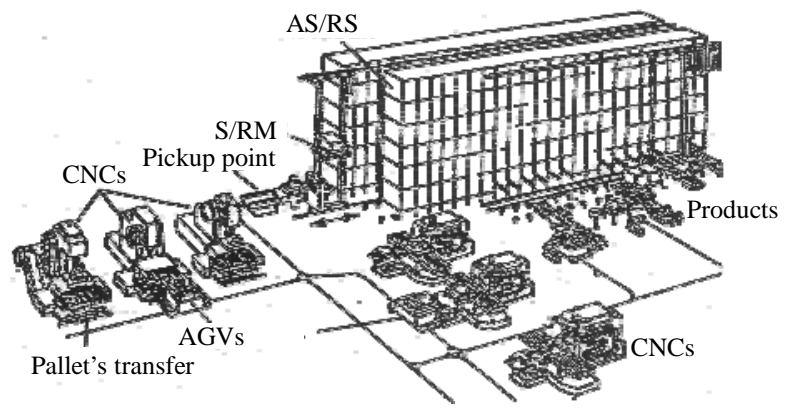

Figure 1. An example of a flexible manufacturing system. 


\subsection{RFID Data Collection in FMS}

RFID is a powerful data collection method which can be used in product control and material handling or other material flow processes [3]. With deployment of RFID systems in FMS, operation control is strengthened with the better visibility of the process. In FMS, pallets, totes or other loads which are flowing through the FMS are tagged with RFID tags. RFID readers read the tags which store the processing information at a pre-assigned location. Sensors (RFID readers) collect the information of material which flows through the processes, and then sends this information into the database. Under certain rules, meaningful events trickle, this could be used to drive Supply Chain Management (SCM) [4], Enterprise Resource Planning (ERP) or Manufacturing Execution System (MES) information systems. Service-oriented architecture (SOA) and event-driven architecture (EDA) are dominant in this field [5,6]. The RFID readers can provide real-time material flow information like probes in the object system. The information includes item information, location, processing time and arrival time with high accuracy. It can be stored in a data stream, into a database or a data warehouse.

For FMS as in Figure 1, RFID readers can be set up at an AS/RS I/O station, a CNC work station and at AGV pickup points. Tags are tagged on pallets and fixtures. When pallets or fixtures flow through the system, readers read the tags and create data records as in Table 1. These records are then processed by RFID middleware. Finally, large volume records will be stored as data streams in an RFID data warehouse [7]. Thus, RFID data becomes the bridge that connects the physical world to the virtual world.

\subsection{Challenges in FMS Simulation}

The environment in which FMS operates is stochastic in nature. So when designing and analyzing FMS, this nature must been taken into consideration. Simulation is a powerful tool to tackle a stochastic situation [8]. There are commercial software packages which can be used to

Table 1. RFID tag records.

\begin{tabular}{ccccc}
\hline Tag ID & $\begin{array}{c}\text { Load } \\
\text { Type }\end{array}$ & Item & $\begin{array}{c}\text { Time } \\
\text { (hh:mm:ss:ms) }\end{array}$ & $\begin{array}{c}\text { Reader } \\
\text { ID }\end{array}$ \\
\hline EE07000001A34621 & pallet & raw1 & $14: 01: 45: 35$ & 1 \\
EE07000001A34622 & pallet & raw2 & $14: 01: 45: 55$ & 2 \\
EF01000001A34621 & fixture & P1 & $14: 05: 05: 55$ & 3 \\
EF01000001A34622 & fixture & P2 & $14: 05: 06: 15$ & 4 \\
EF01000001A34623 & fixture & P3 & $14: 05: 07: 05$ & 5 \\
\hline
\end{tabular}

simulate FMS. But simulation is still a job which needs talented people especially when the objective system is complex. Generally, commercial simulation software packages may alleviate these efforts dramatically, because we can use them to model FMS with the visual entities and components in their Graphical User Interfaces (GUIs), but for invisible data or I/O data, there is not an efficient way to model them. Under many circumstances, a pseudo-random number generator is the only way to drive an FMS simulation.

Random numbers are used in simulations to introduce the variability of the real world into a model. Product inter-arrival times, processing times, time until machine failures, and repair times are examples of events with a duration that varies throughout the operation of a real system. When building a model, decisions must be made how to represent randomly occurring events in the simulation. A popular technique is to attempt to fit estimated or historic real-world data to a distribution. But for FMS, high throughput means a high volume of data. Data analysis is very difficult. On the other hand, so much in-process data is stored in MES or other enterprise information systems, particularly with the deployment of an RFID system. This formal data can be utilized in an FMS simulation.

In the following part of this article, we will discuss FMS simulation using commercial software. A simulation framework using RFID data stream will also be presented.

\section{FMS Simulation Using AutoMod Software}

AutoMod suite is world-leading industrial simulation software, which is provided by Applied Materials, an American company. It has many successful applications in various sectors, such as automobile, semiconductor, aerospace and defense, paper, logistics etc. Many of the top 500 companies in the world have used AutoMod to simulate their production logistics in order to improve the operation and efficiency of departments, reduce inventory and cash flow $[9,10]$.

AutoMod is actually a combination of two programs: a build package and a runtime package. The build package is for physical and logical model definition. After the user has defined the physical and logical components of the model, it is compiled into an executable program, where the simulation and animation run concurrently. The executable model runs very fast and is fully interactive; it can be stopped at any instant in simulated time to view statistics and model status. The latest version of AutoMod provides database I/O functions and a model communications module which supports OPC (OLE for Process Control) or sockets communication. 
The visual components of FMS can be easily modeled with professional AutoMod modules, such as AS/RS, process, conveyor and path-mover subsystem. Without real world data, an FMS model is usually driven by pseudo-random streams or simple sample data which simplify the input or in-process data. Data acquisition codes are embedded in the AutoMod logic files.

\section{Simulation Frameworks Driven by RFID Data Streams}

There are applications in different types of DEVS (Discrete Event Systems) simulations using RFID data. For example, RFID data was used to simulate and analyze hospital operations and resource utilization. From above we know that RFID data can drive the virtual AutoMod model in place of the pseudo-random streams or simple samples. Knowing how to use the RFID data streams collected in the FMS operation period is the problem we need to address. According to different RFID data sources, there are two ways to use RFID data streams in an FMS simulation. An individual framework will be presented under each mode in the following section. Technical details will also be mentioned.

In both these modes, the simulation software concentrates on the operational characters of RFID tags and tagged object name, such as arrival time, arrival location. Other information in the tags is not sent to the software. The transferred information must be formal and can be parsed into meaningful events which can be understood by the software. Transparent standards and protocols must be complied with before the simulation.

\subsection{Offline Mode (Historical RFID Data)}

In offline mode, a simulation model is driven by historical RFID data streams stored in a database. In this mode, processed RFID data has been stored in a database. When an AutoMod model of FMS runs, it reads AutoMod database I/O functions (like Open Database Connectivity function) and parses the RFID data streams into certain events which are meaningful to AutoMod. Figure 2 shows the framework of offline mode.

\subsection{Online Mode (Emulation)}

When RFID data streams are used in online mode, the FMS model interacts with real time RFID data streams. It's a type of hardware-in-loop simulation, or emulation. In this mode, the virtual simulation model is combined with real world equipment and the bridge is the RFID data streams. The whole FMS is divided into two parts. This method is very useful when we want change part of
FMS like a temporary storage warehouse. If an AS/RS is planned to replace the manual high bay warehouse, an AS/RS is built in the AutoMod software. The I/O RFID data source of temporary storage warehouse from the real world RFID readers is used to drive the FMS simulation. So the decision may be made easily with the help of simulation.

The latest AutoMod software provides OPC or sockets communication in its Model Communications Module (MCM, or MCM Plus). Industrial RFID readers always use TCP/IP, OPC or other industrial communication protocols. The framework can be realized as the Figure 3. In this mode, all minor tag reading failures must be ignored as bad data.

\section{Conclusions}

This paper presents different frameworks of FMS simulations using RFID data. Still, there is hard work to be done with respect to the execution especially for reading exceptions. But the framework has been successfully tested. Simulation is a well-proven method to design or analyze FMS. It is valuable to use the data stream to improve the operation of FMS.

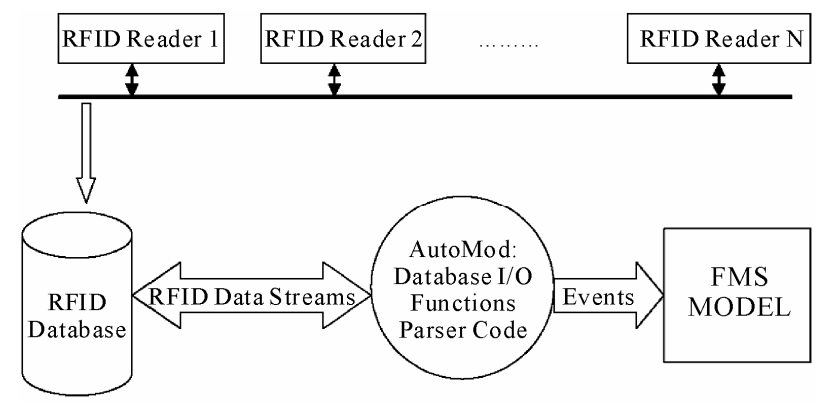

Figure 2. FMS Simulation in Offline Mode; Note: An RFID database stores the preprocessed records from RFID readers (homogeneous or heterogeneous). Database procedures can be executed to get special data view to be used in the simulation.

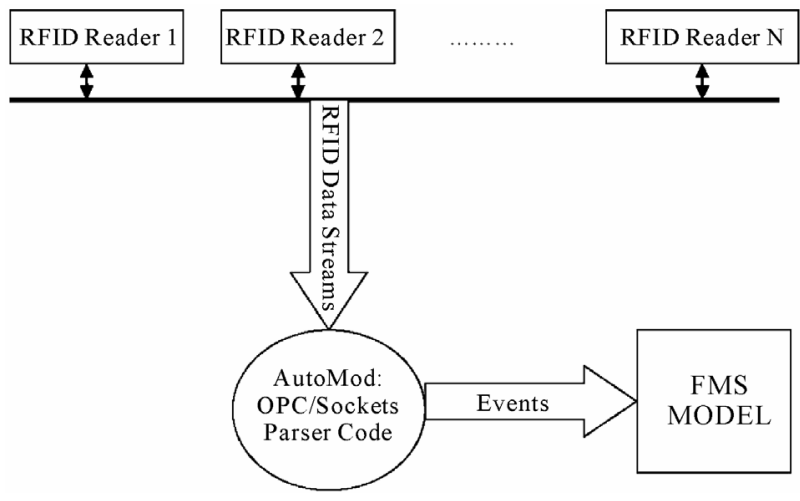

Figure 3. FMS simulation in online mode. 


\section{Acknowledgments}

The work was supported under the research of the National Natural Science Foundation (71071141/ G011203), Doctoral Fund of Ministry of Education of China (20103326110001), Zhejiang Provincial Natural Science Foundation of China (Y6090015, Z1091224), the Contemporary Business and Trade Research Center of Zhejiang Gongshang University (11JDSM02Z).

\section{References}

[1] A. Z. Camdereli, "Misplaced Inventory and Radio-Frequency Identification (RFID) Technology: Information and Coordination," Production and Operations Management, Vol. 19, No. 1, 2010, pp. 1-18. doi:10.1111/j.1937-5956.2009.01057.x

[2] M. Amini, R. F. Otondo, B. D. Janz and M. G. Pitts, "Simulation Modeling and Analysis: A Collateral Application and Exposition of RFID Technology," Production and Operations Management, Vol. 16, No. 5, 2007, pp. 586-598. doi:10.1111/j.1937-5956.2007.tb00282.x

[3] Z.-X. Chen, "The Application of RFID Technology and Logistics," Commercial Research, Vol. 266, No. 6, 2003, pp. 138-142.

[4] H. Amoozad-khalili, R. Tavakkoli-Moghaddam and N. Shahab-Dehkordi, "Influence of Radio Frequency Identification Technology in Logistics, Inventory Control and
Supply China Optimization,” World Academy of Science, Engineering and Technology, Vol. 69, No. 1, 2010, pp. 63-68.

[5] C. Zang and Y. Fan, "Complex Event Processing in Enterprise Information Systems Based on RFID,” Enterprise Information Systems, Vol. 1, No. 1, 2007, pp. 1-3.

[6] O. Gunther, W. Kletti and U. Kubach, "RFID in Manufacturing,” 1st Edition, Springer, Berlin, 2008, pp.1-23.

[7] A. Pradhan, E. Ergen and B. Akinci, “Technological Assessment of Radio Frequency Identification Technology for Indoor Localization,” Journal of Computing in Civil Engineering, Vol. 23, No. 3, 2009, pp. 230-238. doi:10.1061/(ASCE)0887-3801(2009)23:4(230)

[8] Z.-X. Chen and J.-H. Gong, "Current Application Situation and under Certain Rules, Meaningful Events Trickle Development of Logistics Simulation Software,” Journal of Zhejiang Gongshang University, Vol. 85, No. 4, 2007, pp. 29-34.

[9] H.-J. Mao, H.-T. Guo, C. L. Ma, X.-H. Li and J. He, "Modeling and Analysis of Steel Logistics Center Based Automod Simulation Platform," Journal of Southeast University (Natural Science Edition), Vol. 38, No. 2, 2008, pp. 314-318.

[10] Z.-Y. Jiang, H.-F. Kang and X.-C. Huang, "A Platform Non-ferrous Metal Three-Dimensional warehousing Depositing System Simulation Analysis Based on AutoMod Simulation,” Machinery Design \& Manufacture, No. 8, 2010, pp. 258-260. 